FciDoc

International Journal of Anesthesiology \& Research (IJAR)

ISSN 2332-2780

\title{
Serotonin Syndrome Following Methylene Blue Administration for Vasoplegic Syndrome in Heart and Lung Transplantation - A Case Series
}

\author{
Case Report
}

Arias-Morales $C^{1}$, Gonzalez-Zacarias $\mathrm{AA}^{1}$, Essandoh $\mathrm{MK}^{1,2}$, Dimitrova $\mathrm{G}^{1,2}$, Bhatt $\mathrm{AM}^{1,2^{*}}$

${ }^{1}$ Department of Anesthesiology, The Ohio State University Wexner Medical Center, Columbus, Ohio, USA.

${ }^{2}$ Department of Anesthesiology, Division of Cardiothoracic and Vascular Anesthesiology, The Ohio State University Wexner Medical Center, Columbus, Ohio, USA.

\section{Abstract}

Serotonin syndrome is a potentially life threatening adverse drug reaction caused by excessive activation of postsynaptic serotonin receptors in the central nervous system (CNS). It is typically associated with the simultaneous administration of two or more selective serotonin reuptake inhibitors (SSRI) or as a result of a combination with another drug with similar serotonergic activity. The authors describe two cases of intraoperative vasoplegic syndrome necessitating the use of methylene blue $(\mathrm{MB})$ as rescue therapy and subsequently causing serotonin syndrome in the intensive care unit (ICU).

Keywords: Serotonin Syndrome; Vasoplegia; Methylene Blue; Cardiac Surgery; Transplant.

\section{Introduction}

Vasoplegic syndrome (VS) is a well-known form of vasodilatory shock that occurs after cardiac surgery employing cardiopulmonary bypass (CPB) [1]. Treatment of VS is primarily focused on high-dose vasopressor therapy. In addition to vasopressors, methylene blue (MB) proved to be of benefit for refractory VS in various studies [2-4]. The use of MB for VS carries a risk for the development of serotonin syndrome (SS).

SS is a potentially life threatening adverse drug reaction caused by excessive activation of postsynaptic serotonin receptors in the central nervous system (CNS) [5]. The syndrome is typically described as a clinical triad of mental status changes, autonomic instability and neuromuscular abnormalities [6]. It is difficult to diagnose particularly in the intensive care setting due to the potentially wide spectrum of presentation and overall physicians' lack of familiarity with the disease.

The authors describe two cases of intraoperative VS necessitating the use of MB as rescue therapy, and subsequently causing SS in the ICU.

\section{Case 1}

The patient was a 64 year-old female with history of non-ischemic cardiomyopathy and left ventricular assist device (LVAD) in place undergoing orthotopic heart transplantation (OHT). Her comorbidities included atrial fibrillation (AF), monoclonal gammopathy, hypertension (HTN), asthma, osteoporosis, gastroesophageal reflux disease (GERD), and depression for which she was being treated with sertraline. She had recently been upgraded to a United Network for Organ Sharing (UNOS) status level 1A for LVAD malfunction. Following identification of an appropriate donor, she was taken to the operating room. Standard American Society of Anesthesiologists (ASA) monitors were placed.

A pre-induction arterial line was placed. Induction of general anesthesia was uneventful with midazolam, fentanyl, lidocaine, propofol and vecuronium for neuromuscular blockade. Subsequently a 9 French central venous catheter was placed in the right internal jugular vein through which a 7.5 French thermodilution pulmonary artery catheter was placed as standard procedure for OHT at our institution. Following her transplant surgery, she was brought to the ICU with continuous infusions of several vasopressors as well as inotropes for refractory shock. She remained hypotensive despite large doses of vasopressors; therefore $\mathrm{MB}$

\footnotetext{
*Corresponding Author:

Amar M. Bhatt MD,

Department of Anesthesiology, The Ohio State University Wexner Medical Center, 410 W 10th Ave, N411 Doan Hall, Columbus OH, 43235 USA.

Tel: (614) 293-3559

E-mail: Amar.Bhatt@osumc.edu

Received: December 01, 2016

Accepted: December 12, 2016

Published: December 15, 2016

Citation: Arias-Morales C, Gonzalez-Zacarias AA, Essandoh MK, Dimitrova G, Bhatt AM (2016) Serotonin Syndrome Following Methylene Blue Administration for Vasoplegic Syndrome in Heart and Lung Transplantation - A Case Series. Int J Anesth Res. 4(12), 363-367. doi: http://dx.doi.org/10.19070/2332-2780-1600075

Copyright: Bhatt $\mathbf{A M}^{\circ}$ 2016. This is an open-access article distributed under the terms of the Creative Commons Attribution License, which permits unrestricted use, distribution and reproduction in any medium, provided the original author and source are credited.
} 
was used for refractory VS. As the effects of general anesthesia subsided, there were no focal abnormalities recorded in her neurologic evaluation. When ondansetron was administrated for nausea, she progressively developed dilated pupils, ocular clonus, and diffuse muscle rigidity. She subsequently became febrile to 105.7 degrees Fahrenheit, with a bilateral ankle clonus on physical exam.

Delayed malignant hyperthermia and neuroleptic malignant syndrome were clinically ruled out, and serotonin syndrome was suspected. The patient's clinical history as well as pharmacologic treatment including sertraline, MB and ondansetron all implicated $\mathrm{SS}$ as a cause for her abnormal neurologic findings. The purported offending agents were discontinued, and she was treated symptomatically with cyproheptadine, acetaminophen, lorazepam and active cooling. Over the next several hours, her neurologic symptoms improved and her fever subsided. She experienced a full neurologic recovery, and was subsequently discharged home in stable condition.

\section{Case 2}

The patient was a 57 year-old female with history of interstitial lung disease on 5 liters per minute of continuous home oxygen therapy while at rest who presented for bilateral lung transplantation. Her comorbidities included diabetes mellitus type 2, GERD, degenerative disc disease and fibromyalgia for which she was being treated with duloxetine. Following identification of a suitable donor, bilateral lung transplantation was planned, with $\mathrm{CPB}$ as a backup for cardiovascular support.

After placement of standard ASA monitors, a pre-induction arterial line was placed in the left brachial artery. Induction of general anesthesia (GA) was uneventful using midazolam, lidocaine, fentanyl, propofol and rocuronium. Endotracheal intubation was performed with direct laryngoscopy using a Macintosh size 3 blade and a 37 French left-sided double lumen endotracheal tube and position confirmed with fiber optic bronchoscopy. Maintenance of general anesthesia was achieved using inhaled isoflurane as well as intermittent boluses of fentanyl and vecuronium for neuromuscular blockade. A 9 French central venous catheter was placed in the right internal jugular vein through which a continuous cardiac output pulmonary artery catheter placed using pressure waveform guidance. Following reperfusion of the transplanted lungs, the patient developed severe hypotension treated with continuous infusions as well as intermittent boluses of high-dose norepinephrine, vasopressin, and phenylephrine. At this point, due to refractory VS the decision was made to administer MB at a dose of $2 \mathrm{mg} / \mathrm{kg}$. Upon initial rapid administration of approximately half of the dose, the patient developed cardiac conduction abnormalities prompting slowing and eventual discontinuation of the medication. Subsequently, the patient's hemodynamic parameters improved and vasopressor doses were deescalated.

She was transferred to the ICU in stable condition. Several hours later, the patient developed hyperthermia with maximum temperature of 105.7 degrees Fahrenheit; in addition she displayed neurologic findings of muscle rigidity, ankle clonus and pupillary dilation. After ruling out other causes of perioperative hyperthermia, including malignant hyperthermia, as well as other potential neurologic catastrophes, the diagnosis of serotonin syndrome was made. Following the diagnosis, the patient was treated with sup- portive care including active cooling, as well as benzodiazepines for rigidity and clonus; cyproheptadine was considered but was not used due to the resolution of symptoms.

\section{Discussion}

\section{Vasoplegic Syndrome}

VS is a well-known form of vasodilatory shock that occurs in up to $44 \%$ of patients after cardiac surgery employing cardiopulmonary bypass (CPB) [1]. CPB is often associated with a systemic inflammatory response and transient vascular dysfunction that results in profound and persistent vasodilation. A decrease in systemic vascular resistance follows with severe hypotension, refractory to proper fluid resuscitation, and requiring an increased dose of vasopressors despite of high or normal cardiac outputs [7].

Although the exact mechanisms that lead to the development of VS are unknown, it is thought to be related to depletion of natural vasopressors during $\mathrm{CPB}$ [8], excessive complement activation [9], and the development of systemic inflammatory response syndrome (SIRS) $[10,11]$. SIRS post cardiac surgery is believed to be induced by factors such as surgical trauma, changes in body temperature, blood exposure to non-physiologic surfaces, endotoxin release and ischemia-reperfusion of the myocardium $[12,13]$.

As blood passes through the CPB machine, plasma proteins are immediately absorbed to bio-membranes, generating a protein layer that is densely packed and immobile, with some proteins undergoing conformational changes $[14,15]$. The result is the activation of the extrinsic and intrinsic coagulation pathway, the complement, and fibrinolytic pathways. In light of this, VS pathophysiology reflects a series of complex interactions among endothelial cells, plasma proteins, leukocytes, and platelets. Multiple factors including thrombin, $\mathrm{C} 5 \mathrm{a}$ and cytokines, active endothelial cells produce vasoactive substances such as nitric oxide (NO) and prostacyclin that contribute to the persistent hypotension [16].

Blood transfused from the salvage mechanism (cell saver) contribute to the pathogenesis of vasoplegia, because of its hemolyzed erythrocytes, denatured proteins, fat globules and platelet and leukocyte aggregates which potentially stimulate and aggravate inflammatory responses. Additionally, an ischemia-reperfusion syndrome occurs after aortic cross clamp, causing neutrophils to adhere to endothelial cells with the release of free radicals causing direct damage to the tissues, and resulting in increased capillary permeability, interstitial edema and reduced intravascular volume [1].

This inflammatory response caused by CPB generates the formation of fibrin clots and micro emboli that leads to intraoperative myocardial ischemia and elevation of troponin I levels [12]. Nevertheless, the primary challenge in VS patients involves the management of severe hypotension induced by the vasodilatory effect of excessive NO production and decreased plasma levels of arginine vasopressin (AVP) [17]. Low vasopressin (VP) in plasma is attributed to decreased secretion due to an impaired baroreflexmediated VP release, and to excessive secretion of VP with subsequent depletion of pituitary VP stores in the early stages of the inflammatory response $[18,19]$. 
Treatment of VS is primarily focused on high-dose vasopressor therapy. In addition, MB has been used in various studies to show benefit for refractory VS [2-4]. It is thought to function by scavenging free nitric oxide (NO) as well as inhibiting endothelial synthesis of NO [20].

\section{Methylene Blue}

Although the mainstay treatment for VS is the use of high dose vasopressors, $\mathrm{MB}$ is recommended for refractory VS as rescue therapy [4]. MB inhibits vasodilation in different ways. One of the described mechanisms is the inhibition of constitutive and inducible NO synthase [21, 22]. Another mechanism is through the inhibition of guanylyl cyclase which is an important catalyst in vasodilation mediated by NO (Figure 1). MB has been associated with developing SS, especially in patients undergoing cardiothoracic surgery and taking SSRI [23]. MB is a potent and reversible Monoamine Oxidase A (MAO-A) inhibitor, which prevents serotonin metabolism. Several studies have described that MB exerts higher inhibitory effects on MAO-A than MAO-B, probably due to its wider active site $[23,24]$.

SS is rarely described in patients taking a mono-serotonergic therapy. However, this condition may be precipitated by adding a single dose of MB. The Food and Drug Administration (FDA) recommends for patients who are at risk to be exposed to MB to stop taking serotonergic agents at least 2 weeks prior to its potential administration $[25,26]$.

\section{Serotonin Syndrome}

Serotonin Syndrome (SS) is a life-threatening adverse drug reaction caused by the excess of serotonergic activity in the central and peripheral nervous systems [27]. It is typically associated with the simultaneous administration of two or more selective SSRI or as a result of a combination with another drug with similar serotonergic activity. The addition of drugs that inhibit the cytochrome P450 2D6 or 3A4 isoenzymes to an ongoing therapy regimen of an SSRI have been reported [28]. Likewise, different classes of drugs including anesthetics have been implicated in the development of SS. It is important to mention that serotonergic agents may include nonprescription drugs, illicit drugs, and diet supplements (Table1) [27].

Clinically, SS is characterized by a triad of neuromuscular abnormalities (tremor, clonus, myoclonus, and hyperreflexia), autonomic hyperactivity (diaphoresis, fever, tachycardia, tachypnea, and mydriasis), and mental status changes (agitation and confusion) [29]. Interestingly, SS has been reported in all age groups [30]. Symptoms can develop within a few minutes of drug ingestion; however, the most common onset of SS symptoms was reported within 6 to 24 hours from the moment of the purported drug administration [27].

The incidence of SS is certainly unknown. Nonetheless, it is believed that the incidence is rising due to the recent surge of serotoninergic medications prescribed in the clinical setting, specifically with a high predominance in females [30]. Furthermore, SS has been reported in patients with end stage renal disease under treatment with an SSRI in combination with Monoamine Oxidase Inhibitors (MAOi) [28]. Frequently, this condition is undiagnosed or under recognized among physicians and may have serious clinical complications, such as metabolic acidosis, rhabdomyolysis, seizures, renal failure, and disseminated intravascular coagulopathy [31].

SS is entirely a clinical diagnosis, principally based on patient's history and physical examination. Laboratory studies may provide an insight of serious complications or help rule out other diagnoses [30]. Hunter's criteria for serotonin toxicity are the most extensively used for the diagnosis of SS, with a sensitivity of $84 \%$ and a specificity of $97 \%$ [32]. The diagnosis is established if the patient is taking a serotonergic agent and presents any of the following symptoms: spontaneous or inducible clonus plus agitation or diaphoresis, ocular clonus, tremor with hyperreflexia, and hypertonia plus hyperthermia $(>101.3 \mathrm{~F})$ plus ocular clonus or inducible clonus [28], as reported in our two cases.

The differential diagnoses for SS include malignant hyperthermia, neuroleptic malignant syndrome (NMS), and anticholinergic syndrome. Sometimes, SS can be distinguished from other diagnoses by the patients' symptomatology, onset of symptoms, and medication use. Remarkably, patients with SS may experience overlapping symptoms of NMS when causative drugs have been admin-

Figure 1. Inhibition of Nitric Oxide-Mediated Vasodilation by Methylene Blue.

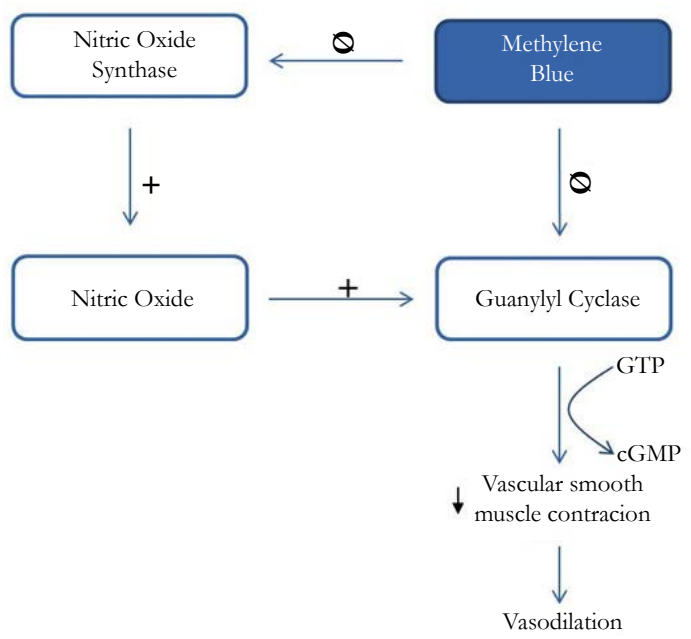


Table 1. Medications Contributing to Serotonin Syndrome.

\begin{tabular}{|c|c|c|}
\hline \multicolumn{3}{|c|}{ Medications Contributing to Serotonin Syndrome } \\
\hline $\begin{array}{l}\text { Antidepressants and Mood Stabilizers } \\
\cdot \text { Selective } \\
\text { Serotonin } \\
\text { Reuptake } \\
\text { Inhibitors } \\
\cdot \text { Serotonin-Norepinephrine } \\
\text { Reuptake } \\
\text { Inhibitors } \\
\cdot \text { Monoamine } \\
\text { Oxidase } \\
\text { Inhibitors } \\
\cdot \text { Serotonin } 2 \mathrm{~A} \\
\text { Receptor } \\
\text { Blockers } \\
\cdot \text { Tricyclic } \\
\text { Antidepressants } \\
\cdot \text { Buspirone } \\
\cdot \text { Lithium } \\
\text { - St. John's Wort }\end{array}$ & $\begin{array}{l}\text { Analgesics } \\
\cdot \text { Cyclobenzaprine } \\
\cdot \text { Fentanyl } \\
\cdot \text { Meperidine } \\
\cdot \text { Oxycodone } \\
\cdot \text { Tramadol } \\
\text { Amphetamines } \\
\cdot \text { Dextroamphetamine } \\
\cdot \text { Methamphetamine } \\
\cdot \text { 3,4 Methylenedioxymethamphetamine } \\
\cdot \text { Sibutramine }\end{array}$ & 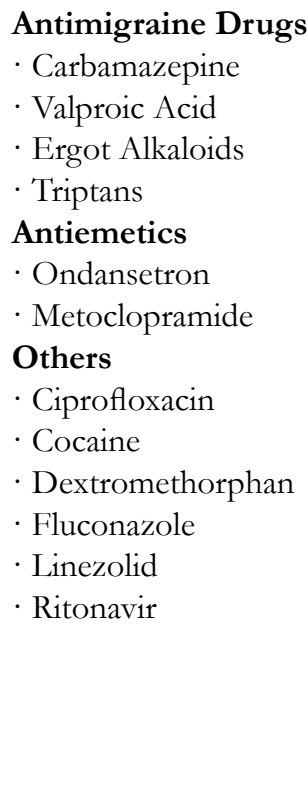 \\
\hline
\end{tabular}

istered simultaneously [33]. For this reason, it is essential to obtain a precise history with patient's comorbidities, history of drugs, dosages with recent additions or changes, as well as substance abuse history. Kotwal et al., published a case report where cocaine was found to contribute to the development of SS [27, 31].

Cyproheptadine, is a first generation anti-histaminic with 5HT2A serotonin antagonist properties, and is the most commonly recommended drug to reverse the symptomatology of SS. Cyproheptadine has a lower incidence of adverse effects and toxicity when compared with chlorpromazine and olanzapine [27, 30]. The recommended initial dose is $12 \mathrm{mg}$, followed by $2 \mathrm{mg}$ every 2 hours if symptoms persist. Once the patient is stabilized, the maintenance dose of $8 \mathrm{mg}$ every 6 hours should be used. Several case reports concluded that cyproheptadine offers symptomatic relief only in mild to moderate cases without interfering with the natural progression of the syndrome, as described in case 1 in the present manuscript. In addition, recent studies have found that high doses of cyproheptadine can be used to prevent and treat hyperthermia, because of their 5HT-2A receptor antagonism [28]. Even though cyproheptadine is widely used, its effectiveness in this life-threatening condition remains poor [27].

Once mild to moderate SS is recognized and treated promptly, the prognosis is generally favorable. As shown in our two case reports, the first-line therapy includes discontinuation of all offending agents with resolution of symptoms within 24 hours [30]. For mild to moderate cases, discontinuation of the serotonergic agents plus hemodynamic supportive care with cardiac monitoring, oxygen therapy, and the administration of benzodiazepines for agitation and tremor may be required. These cases usually resolve within 24 to 72 hours and without the need of hospital admission $[30,34]$. The mainstay of therapy for severe or life-threatening cases includes prompt airway control and intubation, hyperthermia management with active cooling and acetaminophen, treatment of muscle rigidity with neuromuscular blockers, and hemodynamic stabilization with vasopressors or antihypertensive drugs $[27,34,35]$. The administration of serotonin antagonists, cyproheptadine, olanzapine, or chlorpromazine is strongly recommended [27]. Physicians must be aware of SS mostly because of the recent increased of prescribed serotonergic agents in the general population. Thus, obtaining detailed information, early recognition of the symptoms, and prompt treatment management can prevent significant morbidity and mortality $[17,18]$.

\section{Conclusion}

Both of our case reports highlight the complication of VS frequently diagnosed during cardiac and lung transplant surgeries. Although MB is used as a rescue therapy for refractory VS, caution should be used, considering its potent inhibitory effect on monoamine oxidase and associated sequelae. In addition, both transplant patients as well as terminally ill patients are frequently prescribed SSRIs by their primary care physicians putting them at higher risk for the perioperative development of SS. Careful attention to those at increased risk, as well as prompt clinical diagnosis and treatment can be life-saving.

\section{Acknowledgement}

The authors gratefully acknowledge Nicoleta Stoicea, MD, PhD who collaborated with the review and editing of the final manuscript.

\section{References}

[1]. Omar S, A Zedan, K Nugent (2015) Cardiac vasoplegia syndrome: pathophysiology, risk factors and treatment. Am J Med Sci. 349(1): 80-88.

[2]. Levin RL, Bruno GF, Degrange MA, Del Mazo CD, Griotti JJ, et al., (2004) Methylene blue reduces mortality and morbidity in vasoplegic patients after cardiac surgery. The Ann thoracic surg. 77(2): 496-499.

[3]. Leyh RG, Kofidis T, Struber M, Fischer S, Knobloch K, et al., (2003) Methylene blue: the drug of choice for catecholamine-refractory vasoplegia after cardiopulmonary bypass. J thoracic cardiovasc surg. 125(6): 1426-1431.

[4]. Shanmugam G (2005) Vasoplegic syndrome-the role of methylene blue. European journal of cardio-thoracic surgery. 28(5): 705-710.

[5]. Pedavally S, JE Fugate, AA Rabinstein (2014) Serotonin syndrome in the 
intensive care unit: clinical presentations and precipitating medications. Neurocrit care. 21(1): 108-113.

[6]. Boyer EW, M Shannon (2005) The serotonin syndrome. N Engl J Med. 352(11): 1112-1120.

[7]. Özal E, Kuralay E, Yildirim V, Kili S, Bolcal C, et al., (2005) Preoperative methylene blue administration in patients at high risk for vasoplegic syndrome during cardiac surgery. Ann thorac surg. 79(5): 1615-1619.

[8]. Argenziano M, Chen JM, Choudhri AF, Cullinane S, Garfein E, et al., (1998) Management of vasodilatory shock after cardiac surgery: identification of predisposing factors and use of a novel pressor agent. J thoracic cardiovasc surg. 116(6): 973-980.

[9]. Kirklin JK (1991) Prospects for understanding and eliminating the deleterious effects of cardiopulmonary bypass. Ann thorac surg. 51(4): 529-531.

[10]. Wan S, Marchant A, Desmet JM, Antoine M, Zhang H, et al., (1996) Human cytokine responses to cardiac transplantation and coronary artery bypass grafting. J thorac cardiovasc surg. 111(2): 469-477.

[11]. Kerbaul F, C Guidon, PJ LejeuneM Mollo, T Mesana, F Gouin (2002) Hyperprocalcitonemia is related to noninfectious postoperative severe systemic inflammatory response syndrome associated with cardiovascular dysfunction after coronary artery bypass graft surgery. J cardiothoracic vasc anesth. 16(1): 47-53.

[12]. Lasocki S, Provenchere S, Benessiano J, Vicaut E, Lecharny JB, et al., (2002) Cardiac troponin I is an independent predictor of in-hospital death after adult cardiac surgery. Anesthsiology. 97(2): 405-411.

[13]. Cremer J, Martin M, Redl H, Bahrami S, Graeter T, et al., (1996) Systemic inflammatory response syndrome after cardiac operations. Ann Thorac Surg. 61(6): 1714-1720.

[14]. Brash JL, Scott CF, ten Hove P, wojciechowski P, colman RW, et al., (1988) Mechanism of transient adsorption of fibrinogen from plasma to solid surfaces: role of the contact and fibrinolytic systems. Blood. 71(4): 932-939.

[15]. Lindon J, McManama G, Kushner L, Merrill EW, Salzman EW (1986) Does the conformation of adsorbed fibrinogen dictate platelet interactions with artificial surfaces? Blood. 68(2): 355-362.

[16]. Downing SW, LH Edmunds Jr (1992) Release of vasoactive substances during cardiopulmonary bypass. Ann Thorac Surg. 54(6): 1236-43.

[17]. Jochberger S, Velik-Salchner C, Mary VD, Luckner G, Wenzel V, et al., (2009) The vasopressin and copeptin response in patients with vasodilatory shock after cardiac surgery: a prospective, controlled study. Intensive care med. 2009. 35(3): 489-497.

[18]. Reid IA (1997) Role of vasopressin deficiency in the vasodilation of septic shock. Circulation. 95(5): 1108-1110.

[19]. Landry DW, Levin HR, Gallant EM, Ashton RC Jr, Seo S, et al., (1997) Vasopressin deficiency contributes to the vasodilation of septic shock. Circulation. 95(5): 1122-1125.
[20]. Andritsos MJ (2011) Con: Methylene blue should not be used routinely for vasoplegia perioperatively. J cardiothoracic vasc anesth. 25(4): 739-743.

[21]. Klatt P, B Heinzel, M John, M Kastner, E Bohme, et al., (1992) $\mathrm{Ca}^{2+}$ calmodulin-dependent cytochrome $\mathrm{c}$ reductase activity of brain nitric oxide synthase. J Biol Chem. 267(16): 11374-11378.

[22]. Mayer B, F Brunner, K Schmidt (1993) Inhibition of nitric oxide synthesis by methylene blue. Biochem pharmacol. 45(2): 367-374.

[23]. Smith CJ, Wang D, Sqambelluri A, Kramer RS, Gagnon DJ, et al., (2015) Serotonin syndrome following methylene blue administration during cardiothoracic surgery. J Pharm Pract, 2015. 28(2): 207-11.

[24]. Ramsay RR, C Dunford, PK Gillman (2007) Methylene blue and serotonin toxicity: inhibition of monoamine oxidase A (MAO A) confirms a theoretical prediction. Br J Pharmacol. 152(6): 946-51.

[25]. Francescangeli J, S Vaida, AS Bonavia (2016) Perioperative Diagnosis and Treatment of Serotonin Syndrome Following Administration of Methylene Blue. Am J Case Rep. 2016. 17: 347-51.

[26]. Wolvetang T, R Janse, M Ter Horst (2015) Serotonin Syndrome After Methylene Blue Administration During Cardiac Surgery: A Case Report and Review. J Cardiothorac Vasc Anesth. 30(4): 1042-5.

[27]. Ables AZ, R Nagubilli (2010) Prevention, recognition, and management of serotonin syndrome. Am Fam Physician. 81(9): 1139-42.

[28]. Volpi-Abadie J, AM Kaye, AD Kaye (2013) Serotonin syndrome. Ochsner J. 13(4): 533-40.

[29]. Talarico G, Tosto G, Pietracupa S, Piacentini E, Lenzi GL, et al., (2011) Serotonin toxicity: a short review of the literature and two case reports involving citalopram. Neurol Sci. 32(3): 507-9.

[30]. Wilson L, Rooney T, Baugh RF, Millington B (2012) Recognition and management of perioperative serotonin syndrome. Am J Otolaryngol. 33(3): 319-21.

[31]. Kotwal A, SL Cutrona (2015) Serotonin Syndrome in the Setting of Lamotrigine, Aripiprazole, and Cocaine Use. Case Rep Med. 2015: 769531.

[32]. Dunkley EJ, Isbister GK, Sibbritt D, Dawson AH, Whyte IM (2003) The Hunter Serotonin Toxicity Criteria: simple and accurate diagnostic decision rules for serotonin toxicity. OJM. 96(9): 635-642.

[33]. Nisijima K (2015) Serotonin syndrome overlapping with neuroleptic malignant syndrome: A case report and approaches for differentially diagnosing the two syndromes. Asian J Psychiatr. 18: 100-1.

[34]. Adler AR, JA Charnin, SA Quraishi (2015) Serotonin Syndrome: The Potential for a Severe Reaction Between Common Perioperative Medications and Selective Serotonin Reuptake Inhibitors. A A Case Rep. 5(9): 156-9.

[35]. Beakley BD, AM Kaye, AD Kaye (2015) Tramadol, Pharmacology, Side Effects, and Serotonin Syndrome: A Review. Pain Physician. 18(4): 395-400. 